\title{
A linear order and OWA operator for discrete gradual real numbers
}

\author{
Zdenko Takáč \\ Institute of Information Engineering, Automation and Mathematics \\ Faculty of Chemical and Food Technology \\ Slovak University of Technology in Bratislava, Radlinského 9, 81237 Bratislava, Slovak Republic \\ zdenko.takac@stuba.sk
}

\begin{abstract}
In this paper we introduce a class of linear orders for discrete gradual real numbers. Based on the linear orders we propose an OWA operator on the set of discrete gradual numbers and discuss some its properties. This is a first step of our intentions to introduce a class of linear orders, and consequently also OWA operator, for the set of discrete gradual intervals. Because the set of all fuzzy intervals is included in the set of all gradual intervals, proposed linear orders and OWA operators would be applicable in the settings of fuzzy intervals too.
\end{abstract}

Keywords: OWA operator, Ordered weighted averaging operator, Gradual number, Linear order, Total order, Gradual interval, Fuzzy interval

\section{Introduction}

Since Yager in [1] proposed an ordered weighted averaging operator (OWA operator) this operator is one of the most widely used aggregation methods for real numbers. In the recent years there is a growing interest of scholars to study OWA operators for some other objects, e.g. intervals [2], [3] or fuzzy intervals (fuzzy sets) [4], [5]. The obstacle to applying OWA operators on these more difficult structures is the need of linear order on the structures. This is especially significant for fuzzy intervals which are not linearly ordered [6] and whose aggregation is the matter of interest for many authors (e.g. [7], [8], [9], [10], [11], [12], [13]).

A new concept in fuzzy sets theory, namely a gradual element, is discussed in [14] and [15]. This arose from the distinction between fuzziness and imprecision. According to the authors a gradual real number possess fuzziness but not uncertainty. On the other hand fuzzy interval (sometimes called 'fuzzy number') possess both. For the similar point of view on gradualness see [16], [17] and [18].

The aim of this paper is to propose an OWA operator for gradual numbers. The crucial point of this is the existence of a linear order. As is stated in [15] the set of gradual numbers is not linearly ordered. Hence we will consider the discrete case and propose a class of linear orders for discrete gradual real numbers. We give an algorithm for such linear ordering which is the main result of the paper. Based on this linear order we can state a definition of OWA operator for discrete gradual real numbers (DGOWA operator).

This is an introductory paper of our research. In the future work we intend to use these results and propose a linear order and OWA operator (DGIOWA) for discrete gradual intervals (gradual interval is a crisp interval of gradual real numbers). Gradual intervals emerged as a new way of looking at fuzzy intervals [19]: instead of considering them as fuzzy sets, one can see them as crisp intervals of gradual real numbers. Recall that the set of all gradual intervals also include the set of all fuzzy intervals, thus this DGIOWA operator will be applicable to fuzzy intervals too [20].

The paper is organized as follows. Section 2 contains basic definitions and notations that are used in the remaining parts of the paper. In Section 3 a linear order for discrete gradual real numbers is proposed and an algorithm for the linear ordering is given. Section 4 introduces an OWA operator for discrete gradual real numbers and discusses some basic properties of the operator. In Section 5 we briefly discuss our future intentions and conclude the paper.

\section{Preliminaries}

In this section we present some basic concepts and terminology that will be used throughout the paper.

Definition 1 ([14], [15]) A gradual real number $\breve{r}$ is defined by an assignment function $A_{\breve{r}}:(0,1] \rightarrow$ $\mathbb{R}$. The set of all gradual real numbers is denoted by $G(\mathbb{R})$.

For discussion on rationale of gradual numbers see [15]. Hereafter we only consider discrete gradual real numbers.

Definition 2 Let $k$ be a positive integer. A discrete gradual real number $\breve{r}$ is defined by an assignment function $A_{\breve{r}}:\left\{\alpha_{1}, \ldots, \alpha_{k}\right\} \rightarrow \mathbb{R}$, where $0<\alpha_{1}<$ $\alpha_{2}<\ldots<\alpha_{k}=1$. The set of all discrete gradual real numbers is denoted by $D G_{k}(\mathbb{R})$. 
For simplicity we do not distinguish between gradual number and its assignment function, thus we write $\breve{r}(\alpha)$ instead of $A_{\breve{r}}(\alpha)$.

A partial order on discrete gradual real numbers (see [15]) can be defined as follows:

$$
\breve{r} \leq \breve{s} \quad \text { iff } \quad \breve{r}\left(\alpha_{i}\right) \leq \breve{s}\left(\alpha_{i}\right), \forall i \in\{1, \ldots, k\} .
$$

Recall also that the equality is defined in a straightforward way:

$$
\breve{r}=\breve{s} \quad \text { iff } \quad \breve{r}\left(\alpha_{i}\right)=\breve{s}\left(\alpha_{i}\right), \forall i \in\{1, \ldots, k\} .
$$

Clearly, gradual numbers generalize real numbers: for each $r \in \mathbb{R}$ there exists $\breve{r} \in D G_{k}(\mathbb{R})$ such that $\breve{r}\left(\alpha_{i}\right)=r$ for all $i=1, \ldots, k$. We will write $\breve{r} \stackrel{\circ}{=}$ to emphasize that $r$ and $\breve{r}$ are objects of different kinds. For instance, instead of $\breve{r}\left(\alpha_{i}\right)=1$ for all $i=1, \ldots, k$ we will write $\breve{r} \doteq 1$, or simply $\breve{1}$.

Fuzzy intervals, i.e. fuzzy subsets of real line whose $\alpha$-cuts are closed intervals, were closely studied e.g. in [6] or [21]. It is easy to see that fuzzy interval can be described by a pair of gradual numbers (so-called gradual interval) with several properties. See [19] for relation between fuzzy intervals and gradual intervals.

Definition $3([\mathbf{1}])$ Let $\mathbf{w}=\left(w_{1}, \ldots, w_{n}\right) \in[0,1]^{n}$ with $w_{1}+\ldots+w_{n}=1$ be a weighting vector. An ordered weighted averaging operator (OWA for short) associated with $\mathbf{w}$ is a mapping $O W A_{\mathbf{w}}:[0,1]^{n} \rightarrow$ $[0,1]$ defined by

$$
O W A_{\mathbf{w}}\left(x_{1}, \ldots, x_{n}\right)=\sum_{i=1}^{n} w_{i} x_{(i)}
$$

where $x_{(i)}$ denotes the ith largest number among $x_{1}, \ldots, x_{n}$.

Let us emphasize that the input $\left(x_{1}, \ldots, x_{n}\right)$ has to be ordered prior to applying the summation. Hence, if we want to apply an OWA operator, we need a linear order on the relevant set of inputs.

\section{Linear order of discrete gradual real numbers}

In this section we propose a linear order of discrete gradual real numbers. First we give the following algorithm for ranking $\prec$ of discrete gradual real numbers.

Algorithm 1: Let $\breve{r}, \breve{s} \in D G_{k}(\mathbb{R})$ be discrete gradual real numbers, $\mathbf{v}=\left(v_{1}, \ldots, v_{k}\right) \in[0,1]^{k}$ be a weighting vector with $v_{1}+\ldots+v_{k}=1$, and $g$ : $\{0,1, \ldots, k\} \rightarrow[0,1]$ be a function with appropriate properties specified in the algorithm.

\section{Initialization:}

$I=\{1, \ldots, k\}$.

$M:=\emptyset$.

$s:=1$.

For all $i \in I$, let $v_{i}^{\prime}:=v_{i}$.
2. Comparison:

If $\sum_{i \in I} v_{i}^{\prime} \breve{r}\left(\alpha_{i}\right)<\sum_{i \in I} v_{i}^{\prime} \breve{s}\left(\alpha_{i}\right)$, then $\breve{r} \prec \breve{s}$ (END).

If $\sum_{i \in I} v_{i}^{\prime} \breve{r}\left(\alpha_{i}\right)>\sum_{i \in I} v_{i}^{\prime} \breve{s}\left(\alpha_{i}\right)$, then $\breve{s} \prec \breve{r}$ (END).

If $s=1$, then go to step 3 .

If $s=2$, then go to step 4 .

3. Omitting 1:

$M:=M \cup\left\{j \mid v_{j}=\min \left\{v_{i} \mid i \in I\right\}\right\}$.

$I:=I-M$.

If $I \neq \emptyset$, then go to step 5 , else $M:=\emptyset, I=\{1, \ldots, k\}, s:=2$.

4. Omitting 2:

$M:=M \cup \min \left\{j \mid v_{j}=\min \left\{v_{i} \mid i \in I\right\}\right\}$.

$I:=I-M$

If $I=\emptyset$, then $\breve{r}=\breve{s}$ (END).

5. Redistribution of weights:

For all $i \in I$, let $v_{i}^{\prime}:=v_{i}+g(i)$,

(where $g:\{0,1, \ldots, k\} \rightarrow[0,1]$ is a function with $\left.\sum_{i \in I} v_{i}^{\prime}=1\right)$.

Go to step 2.

The intuition behind Algorithm 1 is as follows. Our ranking $\prec$ of discrete gradual real numbers $\breve{r}$ and $\breve{s}$ is based on the usual order (of real numbers) of the fuzzy weighted averages (FWAs for short) of $\breve{r}\left(\alpha_{1}\right), \ldots, \breve{r}\left(\alpha_{k}\right)$ and $\breve{s}\left(\alpha_{1}\right), \ldots, \breve{s}\left(\alpha_{k}\right)$ (step 2 of the algorithm). If the FWAs are equal, we proceed as follows:

- We omit the set of all elements with minimal weight (step 3), redistribute the sum of their weights to the rest of the elements (step 5, we discuss the technique of redistribution below) and compare FWAs again (step 2). We repeat these steps till we get an inequality between FWAs or we omit all the elements.

- If the above procedure does not lead to a satisfactory result (inequality between FWAs), we refine it as follows. We omit the element corresponding to minimal $\alpha_{i}$ among the elements with minimal weight (step 4 ), redistribute its weight to the rest of the elements (step 5) and compare FWAs again (step 2). We repeat these steps till we get an inequality between FWAs or we omit all the elements.

If an inequality between FWAs does not appear through the whole procedure, then it holds $\breve{s}=\breve{r}$ (see Theorem 1).

Redistribution of weights of the omitted elements is done by a redistribution function $g(i)$ in the following way: $v_{i}^{\prime}=v_{i}+g(i)$, for all $i \in I$. The redistribution function $g:\{0,1, \ldots, k\} \rightarrow[0,1]$ should satisfy $\sum_{i \in I} g(i)=\sum_{j \in M} v_{j}$ which clearly forces $\sum_{i \in I} v_{i}^{\prime}=1$ (the sum of all new weights is again equal to 1$)$. 
Example 1. For instance,

$$
g(i)=\frac{v_{s}}{k-|M|}
$$

where $|M|$ denotes the cardinality of $M, v_{s}=$ $\sum_{j \in M} v_{j}$, i.e.

$$
v_{i}^{\prime}=v_{i}+\frac{v_{s}}{k-|M|}
$$

In this case the weight of each element is increased by the same value.

Example 2. Let

$$
g(i)=\frac{v_{i}}{1-v_{s}} \cdot v_{s}
$$

i.e.,

$$
v_{i}^{\prime}=v_{i}+\frac{v_{i}}{1-v_{s}} \cdot v_{s} .
$$

In this case the weights of individual elements are increased in proportion of the original weights.

The following theorem states that the ranking $\prec$ given by Algorithm 1 is a linear order of discrete gradual real numbers provided the weights are nonzero, i.e.,

$$
v_{1}>0, \ldots, v_{k}>0 \text {. }
$$

Moreover, it is not just a single linear order, but a class of linear orders based on the choice of weighting vector $\mathbf{v}$ and redistribution function $g$.

Theorem 1 Let $\prec$ be given by Algorithm 1 for some fixed redistribution function $g$ and weighting vector $\mathbf{v}=\left(v_{1}, \ldots, v_{k}\right) \in(0,1]^{k}$ with $v_{1}+\ldots+v_{k}=$ 1. A binary relation $\preceq_{\mathbf{v}, g}$ defined by

$$
\breve{r} \preceq_{\mathbf{v}, g} \breve{s} \quad \text { iff } \quad(\breve{r} \prec \breve{s} \quad \text { or } \quad \breve{r}=\breve{s})
$$

is a linear order on the set of all discrete gradual real numbers.

Proof. It is obvious that $\preceq_{\mathbf{v}, g}$ is reflexive, transitive and antisymmetric, thus it is a partial order on the set of all gradual real numbers. Hence it is sufficient to show that each pair $\breve{r}, \breve{s}$ of gradual real numbers is comparable by $\preceq_{\mathbf{v}, g}$. From Algorithm 1 we obtain exactly one of the following three results:
1. $\breve{r} \prec \breve{s}$, or
2. $\breve{s} \prec \breve{r}$, or
3 .

$$
\begin{aligned}
\sum_{i \in I_{1}} v_{1 i} \breve{r}\left(\alpha_{i}\right)=\sum_{i \in I_{1}} v_{1 i} \breve{s}\left(\alpha_{i}\right), & I_{1}=\{1,2, \ldots, k\}, \\
\sum_{i \in I_{2}} v_{2 i} \breve{r}\left(\alpha_{i}\right)=\sum_{i \in I_{2}} v_{2 i} \breve{s}\left(\alpha_{i}\right), & \\
\vdots & I_{2} \subseteq I_{1},\left|I_{2}\right|=k-1, \\
\sum_{i \in I_{k}} v_{k i} \breve{r}\left(\alpha_{i}\right)=\sum_{i \in I_{k}} v_{k i} \breve{s}\left(\alpha_{i}\right), & I_{k} \subseteq I_{k-1},\left|I_{k}\right|=1 .
\end{aligned}
$$

From the equalities of item 3 it follows that $\breve{r}\left(\alpha_{i}\right)=\breve{s}\left(\alpha_{i}\right)$ for all $i=1, \ldots, k$, which gives $\breve{r}=\breve{s}$.

It is easy to check that $\preceq_{\mathbf{v}, g}$ refines partial order of gradual real numbers given by (1). This means that, for all weighting vectors $\mathbf{v}$ and redistribution functions $g$ it holds:

$$
\breve{r} \preceq_{\mathbf{v}, g} \breve{s} \quad \text { whenever } \quad \breve{r} \leq \breve{s} \text {. }
$$

Moreover, for each weighting vector $\mathbf{v}$ and redistribution function $g, \preceq \mathbf{v}, g$ generalizes the 'standard' order of real numbers $\leq$, i.e.,

$$
\breve{r} \preceq_{\mathbf{v}, g} \breve{s} \quad \text { iff } \quad r \leq s
$$

for all $r, s \in R$ where $\breve{r} \stackrel{\circ}{=}$ and $\breve{s} \doteq s$.

Example 3. Let weighting vector $\mathbf{v}$ be given by the fifth column of Table 1, discrete gradual real numbers $\breve{r}, \breve{s}$ (see Figure 1 ) by the third and fourth columns, and let redistribution function be $g(i)=$ $\frac{v_{s}}{k-|M|}$. Then $\breve{r} \prec_{\mathbf{v}, g} \breve{s}$. The detailed commentary to application of Algorithm 1 (see Table 1):

- Column 5: For weighting vector $\mathbf{v}$ it holds $F W A(\breve{r})=F W A(\breve{s})=3.23$.

- Step 2 of Algorithm 1.

- Column 6: We omit $\alpha_{1}, \alpha_{2}, \alpha_{3}, \alpha_{4}$, i.e., $M=$ $\{1,2,3,4\}$ and redistribute the weights. Then $F W A(\breve{r})=F W A(\breve{s})=3.44$.

- Steps 3, 5 and 2 of Algorithm 1.

- Column 7: We also omit $\alpha_{5}, \alpha_{6}, \alpha_{7}$, i.e., $M=$ $\{1,2, \ldots, 7\}$ and redistribute the weights. Then $F W A(\breve{r})=F W A(\breve{s})=3$.

- Steps 3, 5 and 2 of Algorithm 1.

- Column 8: We should also omit $\alpha_{8}, \alpha_{9}, \alpha_{10}$, i.e., $M=\{1,2, \ldots, 10\}$ and $I=\emptyset$. This is the end of Omitting 1 , we let $M:=\emptyset, I=\{1, \ldots, k\}$, $s:=2$ and start Omitting 2. First we omit $\alpha_{1}$, i.e., $M=\{1\}$ and redistribute the weights. Then $F W A(\breve{r})=F W A(\breve{s})=3.24 \overline{5}$.

- Steps 3, 4, 5 and 2 of Algorithm 1.

- Column 9: We also omit $\alpha_{2}$, i.e., $M=\{1,2\}$ and redistribute the weights. Then $F W A(\breve{r})=$ $F W A(\breve{s})=3.2525$.

- Steps 4, 5 and 2 of Algorithm 1.

- Column 10: We also omit $\alpha_{3}$, i.e., $M=\{1,2,3\}$ and redistribute the weights. Then $F W A(\breve{r})=$ $3.39 \leq F W A(\breve{s})=3.49$. Thus $\breve{r} \prec_{\mathbf{v}, g} \breve{s}$.

- Steps 4, 5 and 2 (END) of Algorithm 1.

Remark 1. Although gradual numbers are functions from $(0.1]$ to real numbers, we depict them in a reversed form, i.e. independent variable $\alpha_{i} \in$ $(0,1]$ is on the vertical axis and dependent variables $\breve{r}\left(\alpha_{i}\right), \breve{s}\left(\alpha_{i}\right)$ are on the horizontal axis. Note that we work with discrete gradual numbers, hence only the points are important and the lines which connect the points are added just for better lucidity. 


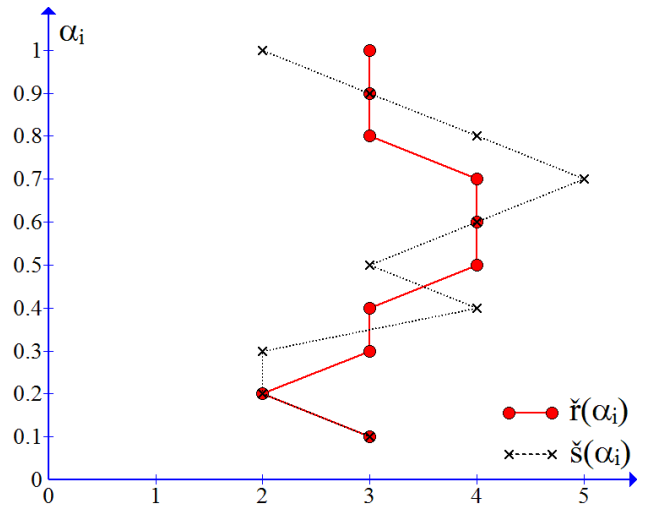

Figure 1: Discrete gradual real numbers $\breve{r}$ and $\breve{s}$ given by Table 1 from Example 3 . See Remark 1 for discussion on graphical representation of discrete gradual real numbers.

It is routine exercise to check that the linear order $\preceq_{\mathbf{v}, g}$ depends on weighting vector $\mathbf{v}$. By the following example we will show that it also depends on redistribution function $g$.

Example 4. Let weighting vector $\mathbf{v}$ be given by the fifth column of Table 2, discrete gradual real numbers $\breve{r}, \breve{s}$ by the third and fourth columns, and let redistribution functions be $g_{1}(i)=\frac{v_{i}}{1-v_{s}} \cdot v_{s}$ (the algorithm is described by the sixth and seventh columns) and $g_{2}(i)=\frac{v_{s}}{k-|M|}$ (the algorithm is described by the eighth column). Then $\breve{s} \prec_{\mathbf{v}, g_{1}} \breve{r}$ and $\breve{r} \prec_{\mathbf{v}, g_{2}} \breve{s}$ (see Table 2).

\section{OWA operators for discrete gradual real numbers}

In this section an OWA operator for discrete gradual real numbers is introduced and some of its basic properties are discussed. Note that this operator is based on the linear order proposed in the previous section.

Definition 4 Let $\breve{\mathbf{w}}=\left(\breve{w}_{1}, \ldots, \breve{w}_{n}\right) \quad \in$ $D G_{k}([0,1])^{n}$ with $\breve{w}_{1}+\ldots+\breve{w}_{n}=\breve{1}$ be $a$ weighting vector of discrete gradual numbers. $A$ discrete gradual numbers OWA operator (DGOWA for short) associated with $\breve{\mathbf{w}}$ is a mapping $D G O W A_{\breve{\mathbf{w}}, g}^{\sqsupset}: D G_{k}(\mathbb{R})^{n} \rightarrow D G_{k}(\mathbb{R})$ defined by

$$
\operatorname{DGOW} A_{\breve{\mathbf{w}}}^{\preceq \mathbf{v}, g}\left(\breve{r}_{1}, \ldots, \breve{r}_{n}\right)=\sum_{i=1}^{n} \breve{w}_{i} \breve{r}_{(i)},
$$

where $\breve{r}_{(i)}, i=1, \ldots, n$, denote the ith greatest component of the input $\left(\breve{r}_{1}, \ldots, \breve{r}_{n}\right)$ with respect to a linear order $\preceq_{\mathbf{v}, g}$.

We used discrete gradual real numbers as a weighting vector in the definition of DGOWA, which is more general approach than using real weights. Now we show that DGOWA operator satisfies the basic properties of aggregation operators [22], [23], [24].

Theorem 2 For any $D G O W A_{\breve{\mathbf{w}}}^{\preceq \mathbf{v} g}$ on $D G_{k}([0,1])$ it holds:

1. $D G O W A_{\breve{\mathbf{w}}}^{\preceq \mathbf{v}, g}(\breve{0}, \ldots, \breve{0})=\breve{0}$

2. $D G O W A_{\breve{\mathbf{w}}}^{\preceq \mathbf{v}, g}(\breve{1}, \ldots, \breve{1})=\breve{1}$

3. $\breve{r}_{1} \leq \breve{s}_{1}, \ldots, \breve{r}_{n} \leq \breve{s}_{n}$ imply $\operatorname{DGOW} A_{\breve{\mathbf{w}}, g}^{\breve{v}_{1}}\left(\breve{r}_{1}, \ldots, \breve{r}_{n}\right) \leq$ $D G O W A_{\breve{\mathbf{w}}}^{-} \mathbf{v},\left(\breve{s}_{1}, \ldots, \breve{s}_{n}\right)$ for all $\breve{r}_{1}, \ldots, \breve{r}_{n}, \breve{s}_{1}, \ldots, \breve{s}_{n} \in D G_{k}([0,1])$.

Proof. The proof is straightforward and therefore omitted.

The calculation of DGOWA via the gradual number arithmetic [15] is simple. Equation (2) can be replaced by the paralel calculation of $k$ independent results:

$$
\operatorname{DGOW} A_{\breve{\mathbf{w}}, g}^{\preceq}\left(\breve{r}_{1}, \ldots, \breve{r}_{n}\right)\left(\alpha_{j}\right)=\sum_{i=1}^{n} \breve{w}_{i}\left(\alpha_{j}\right) \breve{r}_{(i)}\left(\alpha_{j}\right)
$$

for all $j=1, \ldots, k$. This means that the most computational cost is due to ordering of the input $\left(\breve{r}_{1}, \ldots, \breve{r}_{n}\right)$ with respect to a linear order $\preceq_{\mathbf{v}, g}$.

Using DGOWA for real numbers and real weights leads to the same results as 'standard' OWA operator, hence, the proposed DGOWA operator for discrete gradual real numbers encompasses 'standard' OWA operator for real numbers (see the following theorem).

Theorem 3 Let $\mathbf{w}=\left(w_{1}, \ldots, w_{n}\right) \in[0,1]^{n}$ satisfy $w_{1}+\ldots+w_{n}=1$, and $r_{1}, \ldots, r_{n} \in \mathbb{R}$. Let $\breve{w}_{i} \stackrel{\circ}{=} w_{i}$, $\breve{r}_{i} \stackrel{\circ}{=} r_{i}$, for $i=1, \ldots, n$, be discrete gradual real numbers, and $\breve{\mathbf{w}}=\left(\breve{w}_{1}, \ldots, \breve{w}_{n}\right)$. Then

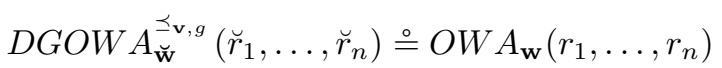

for any weighting vector $\mathbf{v}$ and redistribution function $g$.

Proof. It is easy to check that $r_{(n)} \leq \ldots \leq r_{(1)}$ implies $\breve{r}_{(n)} \preceq_{\mathbf{v}, g} \ldots \preceq_{\mathbf{v}, g} \breve{r}_{(1)}$ for any $\mathbf{v}$ and $g$. Hence

$$
\begin{aligned}
& \operatorname{DGOW} A_{\breve{\mathbf{w}}}^{\breve{v}}\left(\breve{r}_{1}, \ldots, \breve{r}_{n}\right)=\sum_{i=1}^{n} \breve{w}_{i} \breve{r}_{(i)} \stackrel{\circ}{=} \\
& \stackrel{\circ}{=} \sum_{i=1}^{n} w_{i} r_{(i)}=O W A_{\mathbf{w}}\left(r_{1}, \ldots, r_{n}\right) \text {. }
\end{aligned}
$$

Although equation (3) shows that DGOWA can be calculated as $k$ independent results for each $\alpha_{i}$, $i=1, \ldots, k$, it is worth pointing out that in general DGOWA operator cannot be represented by $k$ 'standard' OWA operators for real numbers. In other words, it is not true that:

$$
\begin{array}{r}
D G O W A_{\left(\breve{w}_{1}, \ldots, \breve{w}_{n}\right)}^{\mathfrak{v}_{\mathbf{v}}}\left(\breve{r}_{1}, \ldots, \breve{r}_{n}\right)\left(\alpha_{j}\right)= \\
=O W A_{\left(\breve{w}_{1}\left(\alpha_{j}\right), \ldots, \breve{w}_{n}\left(\alpha_{j}\right)\right)}\left(\breve{r}_{1}\left(\alpha_{j}\right), \ldots, \breve{r}_{n}\left(\alpha_{j}\right)\right)
\end{array}
$$


for all $\mathbf{v}, g$ and $j=1, \ldots, k$.

This is an important fact which shows that our approach takes into account the specific nature of real gradual numbers. So the using of real gradual numbers is not equal to just repeatedly using of real numbers. The reason is as follows: the $i$ th greatest element of $\breve{r}_{1}, \ldots, \breve{r}_{n}$ with respect to $\preceq_{\mathbf{v}, g}$ needs not correspond to the $i$ th greatest element of $\breve{r}_{1}\left(\alpha_{j}\right), \ldots, \breve{r}_{n}\left(\alpha_{j}\right)$ with respect to the 'standard' order of real numbers (see the following counterexample).

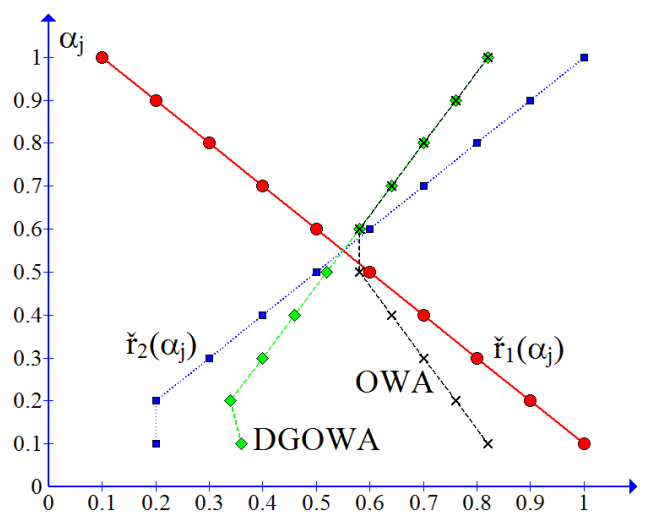

Figure 2: Discrete gradual real numbers $\breve{r}_{1}$ and $\breve{r}_{2}$ given by Table 3 . We can see that, for all $j \in\{1, \ldots, 5\}, D G O W A_{(0.8,0.2)}^{\Upsilon_{\mathbf{v}, g}}\left(\breve{r}_{1}, \breve{r}_{2}\right)$ is not equal to $O W A_{(0.8,0.2)}\left(\breve{r}_{1}\left(\alpha_{j}\right), \breve{r}_{2}\left(\alpha_{j}\right)\right)$. See Example 5 .

Example 5. Let discrete gradual real numbers $\breve{r}_{1}, \breve{r}_{2}$ be given by Table 3, see Figure 2. Clearly, $\breve{r}_{1} \preceq_{\mathbf{v}, g}{\breve{r_{2}}}_{\text {for }} v=(0.1,0.1, \ldots, 0.1)$. We can see that $D G O W A_{(0.8,0.2)}^{-\checkmark v, g}\left(\breve{r}_{1}, \breve{r}_{2}\right)\left(\alpha_{j}\right)$ is not equal to $O W A_{(0.8,0.2)}\left(\breve{r}_{1}\left(\alpha_{j}\right), \breve{r}_{2}\left(\alpha_{j}\right)\right)$ for $j \in\{1, \ldots, 5\}$. The difference lays in the fact that in DGOWA the greater weight 0.8 is assigned to the greater element $\breve{r}_{2}$ for all $\alpha_{j}$, however, in OWA operator the greater weight is assigned to the greater number of the pair $\breve{r}_{1}\left(\alpha_{j}\right), \breve{r}_{2}\left(\alpha_{j}\right)$ which is $\breve{r}_{1}\left(\alpha_{j}\right)$ for $j=1, \ldots, 5$, and $\breve{r}_{2}\left(\alpha_{j}\right)$ for $j=6, \ldots, 10$. This means that

$$
\begin{gathered}
\operatorname{DGOW} A_{(0.8,0.2)}^{-\mathbf{v}, g}\left(\breve{r}_{1}, \breve{r}_{2}\right)\left(\alpha_{j}\right)= \\
=0.8 \cdot \breve{r}_{2}\left(\alpha_{j}\right)+0.2 \cdot \breve{r}_{1}\left(\alpha_{j}\right)
\end{gathered}
$$

and

$$
\begin{gathered}
O W A_{(0.8,0.2)}\left(\breve{r}_{1}\left(\alpha_{j}\right), \breve{r}_{2}\left(\alpha_{j}\right)\right)= \\
=0.8 \max \left\{\breve{r}_{1}\left(\alpha_{j}\right), \breve{r}_{2}\left(\alpha_{j}\right)\right\}+0.2 \min \left\{\breve{r}_{1}\left(\alpha_{j}\right), \breve{r}_{2}\left(\alpha_{j}\right)\right\} .
\end{gathered}
$$

For complete results see Table 3 and Figure 2. Recall that the difference disappears if $\breve{r}_{1} \leq \breve{r_{2}}$.

\section{Conclusion}

We introduced a class of linear orders for discrete gradual real numbers, and consequently proposed an DGOWA operator which is an OWA operator on the set of discrete gradual numbers. Although the calculation of DGOWA operator can be done independently for each level $\alpha$, we showed that DGOWA operator cannot be represented by $k$ OWA operators for real numbers.

In [19] gradual intervals were defined as ordered pairs of gradual real numbers $[\breve{a}, \breve{b}]$ where $\breve{a} \leq \breve{b}$. According to the authors of [19] this is a natural way of looking at fuzzy intervals. Moreover, the notion of gradual interval is more general than that of fuzzy interval. Hence our future intentions are the following. Once we have linear order for discrete real gradual numbers, we can propose a linear order for discrete gradual intervals. Consequently, we can introduce an OWA operator for discrete gradual intervals too. Because gradual intervals encompass fuzzy intervals, the resulting OWA operator would also be applicable to fuzzy intervals with discretized range which is very important (the need for OWA operators on fuzzy intervals is presented e.g. in [4] and $[5])$.

\section{Acknowledgement}

This work was partially supported by the Grant VEGA 1/0419/13.

\section{References}

[1] R.R. Yager. On ordered weighted averaging aggregation operators in multicriteria decisionmaking. IEEE Transactions on Systems Man and Cybernetics, 18(1):183-190, Jan 1988.

[2] H. Bustince, M. Galar, B. Bedregal, A. Kolesárová, and R. Mesiar. A new approach to interval-valued choquet integrals and the problem of ordering in interval-valued fuzzy set applications. IEEE Transactions on Fuzzy Systems, 21(6):1150-1162, 2013.

[3] Z.S. Xu and Q.L. Da. The uncertain OWA operator. International Journal of Intelligent Systems, 17(6):569-575, 2002.

[4] S.-M. Zhou, F. Chiclana, R.I. John, and J.M. Garibaldi. Type-1 OWA operators for aggregating uncertain information with uncertain weights induced by type-2 linguistic quantifiers. Fuzzy Sets and Systems, 159(24):32813296, 2008.

[5] S.-M. Zhou, F. Chiclana, R.I John, and J.M. Garibaldi. Alpha-level aggregation: A practical approach to type-1 OWA operation for aggregating uncertain information with applications to breast cancer treatments. IEEE Transactions on Knowledge and Data Engineering, 23(10):1455-1468, 2011.

[6] Didier Dubois, Etienne Kerre, Radko Mesiar, and Henri Prade. Fuzzy interval analysis. In Didier Dubois and Henri Prade, editors, Fundamentals of Fuzzy Sets, volume 7 of The Handbooks of Fuzzy Sets Series, pages 483-581. Springer US, 2000. 
[7] Zsolt Gera and József Dombi. Exact calculations of extended logical operations on fuzzy truth values. Fuzzy Sets and Systems, 159(11):1309-1326, 2008.

[8] Bao Qing Hu and C.K. Kwong. On type-2 fuzzy sets and their t-norm operations. Information Sciences, 255(0):58 - 81, 2014.

[9] G. Mayor, A.R. Soto, J. Suner, and E. Trillas. Multi-dimensional aggregation of fuzzy numbers through the extension principle. In Data mining, Rough sets and Granular computing, volume 95 of Studies in Fuzziness and Soft Computing, 2002, pages 350-363, 2002.

[10] J.T. Starczewski. Extended triangular norms. Information Sciences, 179(6):742-757, 2009.

[11] Z. Takáč. Aggregation of fuzzy truth values. Information Sciences, 271:1-13, 2014.

[12] A. Takači. General aggregation operators acting on fuzzy numbers induced by ordinary aggregation operators. Novi Sad Journal of Mathematics, 33(2):67-76, 2003.

[13] Chun Yong Wang. Notes on aggregation of fuzzy truth values. Information Sciences, 296(0):119 - 127, 2015.

[14] D. Dubois and H. Prade. Gradual elements in a fuzzy set. Soft Computing, 12(2):165-175, 2008.

[15] J. Fortin, D. Dubois, and H. Fargier. Gradual numbers and their application to fuzzy interval analysis. IEEE Transactions on Fuzzy Systems, 16(2):388-402, 2008.

[16] D. Sánchez, M. Delgado, M.A. Vila, and J. Chamorro-Martínez. On a non-nested levelbased representation of fuzziness. Fuzzy Sets and Systems, 192:159-175, 2012.

[17] W. Kosiński, P. Prokopowicz, and A. Rosa. Defuzzification functionals of ordered fuzzy numbers. IEEE Transactions on Fuzzy Systems, 21(6):1163-1169, 2013.

[18] T.P. Martin and B. Azvine. The X-mu approach: Fuzzy quantities, fuzzy arithmetic and fuzzy association rules. In IEEE Symposium on Foundations of Computational Intelligence (FOCI), 2013, pages 24-29, April 2013.

[19] W.A Lodwick and E.A Untiedt. A comparison of interval analysis using constraint interval arithmetic and fuzzy interval analysis using gradual numbers. In Fuzzy Information Processing Society, 2008. NAFIPS 2008. Annual Meeting of the North American, pages 16, May 2008.

[20] Z. Takáč. Owa operator for discrete gradual numbers and gradual intervals: implications to fuzzy intervals and multi-expert decision making. Kybernetika, 2015, to appear.

[21] Didier Dubois and Henri Prade. Fuzzy Sets and Systems: Theory and Applications. Academic Press, New York, 1980.

[22] G. Beliakov, A. Pradera, and T. Calvo. Aggregation Functions: A Guide for Practitioners.
Studies in Fuzziness and Soft Computing, Volume 221, 2007.

[23] M. Grabisch, J.L. Marichal, R. Mesiar, and E. Pap. Aggregation Functions. Cambridge University Press, Cambridge, 2009.

[24] R. Mesiar, A. Kolesárová, T. Calvo, and M. Komorníková. A review of aggregation functions. In Fuzzy Sets and Their Extensions: Representation, Aggregation and Models, H. Bustince et al. (Eds.), Springer, Berlin, 2008, pages 121144, 2008. 


\begin{tabular}{c|c|cc|c|ccccc}
$i$ & $\alpha_{i}$ & $\breve{r}\left(\alpha_{i}\right)$ & $\breve{s}\left(\alpha_{i}\right)$ & $v_{i}$ & $v_{i}^{\prime}$ & $v_{i}^{\prime}$ & $v_{i}^{\prime}$ & $v_{i}^{\prime}$ & $v_{i}^{\prime}$ \\
\hline 10 & 1 & 3 & 2 & 0.14 & $0.18 \overline{6}$ & $0 . \overline{3}$ & $0.14 \overline{7}$ & 0.1575 & 0.17 \\
9 & 0.9 & 3 & 3 & 0.14 & $0.18 \overline{6}$ & $0 . \overline{3}$ & $0.14 \overline{7}$ & 0.1575 & 0.17 \\
8 & 0.8 & 3 & 4 & 0.14 & $0.18 \overline{6}$ & $0 . \overline{3}$ & $0.14 \overline{7}$ & 0.1575 & 0.17 \\
7 & 0.7 & 4 & 5 & 0.1 & $0.14 \overline{6}$ & & $0.10 \overline{7}$ & 0.1175 & 0.13 \\
6 & 0.6 & 4 & 4 & 0.1 & $0.14 \overline{6}$ & & $0.10 \overline{7}$ & 0.1175 & 0.13 \\
5 & 0.5 & 4 & 3 & 0.1 & $0.14 \overline{6}$ & & $0.10 \overline{7}$ & 0.1175 & 0.13 \\
4 & 0.4 & 3 & 4 & 0.07 & & & $0.0 \overline{7}$ & 0.0875 & 0.1 \\
3 & 0.3 & 3 & 2 & 0.07 & & & $0.0 \overline{\overline{7}}$ & 0.0875 & \\
2 & 0.2 & 2 & 2 & 0.07 & & & $0.0 \overline{7}$ & & \\
1 & 0.1 & 3 & 3 & 0.07 & & & & & \\
\hline$F W A(\breve{r})$ & & & & 3.23 & 3.44 & 3 & $3.24 \overline{5}$ & 3.3525 & $\mathbf{3 . 3 9}$ \\
$F W A(\breve{s})$ & & & & 3.23 & 3.44 & 3 & $3.24 \overline{5}$ & 3.3525 & $\mathbf{3 . 4 9}$ \\
order & & & & $?$ & $?$ & $?$ & $?$ & $?$ & $\breve{r} \prec \mathbf{v}, g$
\end{tabular}

Table 1: Application of Algorithm 1 (see the detailed commentary in Example 3).

\begin{tabular}{c|c|cc|c|cc|c}
$i$ & $\alpha_{i}$ & $\breve{r}\left(\alpha_{i}\right)$ & $\breve{s}\left(\alpha_{i}\right)$ & $v_{i}$ & $v_{i, g_{1}}^{\prime}$ & $v_{i, g_{1}}^{\prime}$ & $v_{i, g_{2}}^{\prime}$ \\
\hline 10 & 1 & 5 & 2 & 0.14 & 0.1591 & 0.1944 & 0.155 \\
9 & 0.9 & 5 & 2 & 0.14 & 0.1591 & 0.1944 & 0.155 \\
8 & 0.8 & 4 & 3 & 0.12 & 0.1364 & $0.1 \overline{6}$ & 0.135 \\
7 & 0.7 & 4 & 3 & 0.12 & 0.1364 & $0.1 \overline{6}$ & 0.135 \\
6 & 0.6 & 3 & 5 & 0.1 & 0.1136 & 0.1389 & 0.115 \\
5 & 0.5 & 2 & 6 & 0.1 & 0.1136 & 0.1389 & 0.115 \\
4 & 0.4 & 2 & 5 & 0.08 & 0.0909 & & 0.095 \\
3 & 0.3 & 2 & 5 & 0.08 & 0.0909 & & 0.095 \\
2 & 0.2 & 3 & 4 & 0.06 & & & \\
1 & 0.1 & 3 & 2 & 0.06 & & & \\
\hline$F W A(\breve{r})$ & & & & 3.54 & 3.6136 & $\mathbf{3 . 9 7 2 2}$ & $\mathbf{3 . 5 8 5}$ \\
$F W A(\breve{s})$ & & & & 3.54 & 3.6136 & $\mathbf{3 . 3 0 5 6}$ & $\mathbf{3 . 6 4 5}$ \\
order & & & & $?$ & $?$ & $\breve{s} \prec \mathbf{v}, g_{1} \breve{r}$ & $\breve{r} \prec \mathbf{v}, g_{2} \breve{s}$
\end{tabular}

Table 2: Application of Algorithm 1 (Example 4). By $v_{i, g_{1}}^{\prime}$ and $v_{i, g_{2}}^{\prime}$ are denoted weights calculated via redistribution functions $g_{1}$ and $g_{2}$, respectively.

\begin{tabular}{c|c|cc|c|c}
$j$ & $\alpha_{j}$ & $\breve{r}_{1}\left(\alpha_{j}\right)$ & $\breve{r}_{2}\left(\alpha_{j}\right)$ & $D G O W A$ & $O W A$ \\
\hline 10 & 1 & 0.1 & 1 & 0.82 & 0.82 \\
9 & 0.9 & 0.2 & 0.9 & 0.76 & 0.76 \\
8 & 0.8 & 0.3 & 0.8 & 0.7 & 0.7 \\
7 & 0.7 & 0.4 & 0.7 & 0.64 & 0.64 \\
6 & 0.6 & 0.5 & 0.6 & 0.58 & 0.58 \\
5 & 0.5 & 0.6 & 0.5 & 0.52 & 0.58 \\
4 & 0.4 & 0.7 & 0.4 & 0.46 & 0.64 \\
3 & 0.3 & 0.8 & 0.3 & 0.4 & 0.7 \\
2 & 0.2 & 0.9 & 0.2 & 0.34 & 0.76 \\
1 & 0.1 & 1 & 0.2 & 0.36 & 0.84
\end{tabular}

Table 3: A counterexample to equation (4). See Example 5. 